\title{
Sonographically Guided Percutaneous Needle Tenotomy for the Treatment of Chronic Tendinosis
}

\author{
Jeffrey A. Housner, MD, Jon A. Jacobson, MD, \\ Roberta Misko, PT, PA-C
}

\begin{abstract}
Objective. Initial reports have shown percutaneous sonographically guided needle tenotomy without corticosteroid injection to be effective for the treatment of tennis elbow. The purpose of this study was to determine the effectiveness of this procedure with various tendons throughout the body. Methods. Fourteen tendons in 13 patients were identified as having a greater than 6-month history of clinical presentation consistent with tendinopathy that had failed treatment with physical therapy. All patients were treated with sonographically guided percutaneous tenotomy using a 22-gauge needle and a local anesthetic. A visual analog scale (VAS) pain score measurement was obtained before the procedure and at 4- and 12-week follow-up appointments. All complications were recorded. Results. The 14 tendons in this study included patellar (5), Achilles (4), proximal gluteus medius (1), proximal iliotibial tract (1), proximal hamstring (1), common extensor elbow (1), and proximal rectus femoris (1). The composite VAS score was significantly lower at both 4 weeks (mean \pm SEM, $2.4 \pm 0.7)$ and 12 weeks $(2.2$ $\pm 0.7)$ compared with the baseline $(5.8 \pm 0.6 ; P<.001)$. No complications or morbidity occurred. Conclusions. Sonographically guided percutaneous tenotomy of tendinosis was effective in improving patient symptoms without complications. Further investigation with additional patients and comparisons to alternative treatments is needed to validate these preliminary results. Key words: sonographic guidance; tendinopathy; tendinosis; tenotomy; treatment.
\end{abstract}

Abbreviations

VAS, visual analog scale

Received March 4, 2009, from the Department of Orthopedic Surgery, University of Michigan MedSport, Ann Arbor, Michigan USA (J.A.H., R.M.); and Departments of Radiology (J.A.J.) and Otolaryngology (R.M.), University of Michigan, Ann Arbor, Michigan USA. Revision requested March 9, 2009. Revised manuscript accepted for publication April 14, 2009.

Address correspondence to Jeffrey A. Housner, MD, Department of Orthopedic Surgery, University of Michigan MedSport, 24 Frank Lloyd Wright Dr, Ann Arbor, MI 48105-9755 USA.

E-mail: jhousner@umich.edu

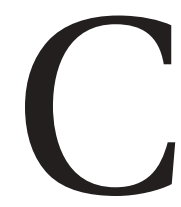

hronic tendon injuries resulting in tendinosis (or tendinopathy) are common problems encountered in musculoskeletal medicine. Conventional nonsurgical treatment options typically recommended include relative rest, cryotherapy, nonsteroidal anti-inflammatory medications, physical therapy (stretching, eccentric strengthening, and modalities), and biomechanical devices (braces and orthoses)..$^{1}$ Surgical intervention has been reported as an additional treatment option in those cases in which conservative treatment has failed..$^{2-5}$ Reported surgical success rates have been variable, ${ }^{2,3,5}$ with undesirable complication rates ${ }^{6}$ and prolonged recovery. ${ }^{7,8}$ 
In an effort to shorten recovery and reduce morbidity, less invasive approaches to the treatment of chronic tendon injuries have been studied and shown to be effective. Early studies have described a minimally invasive longitudinal tenotomy procedure using a scalpel blade and sonographic guidance for the treatment of chronic Achilles and patellar tendinopathy, showing decreased morbidity and outcomes comparable with those of more extensive "open" procedures. ${ }^{9,10}$ More recently, there has been investigation into novel treatments such as sonographically guided extracorporeal shock wave therapy ${ }^{11-13}$ and radio frequency-based microtenotomy. ${ }^{14}$ McShane et $\mathrm{al}^{15}$ have published their results of sonographically guided needle tenotomy with corticosteroid injection for treatment of common extensor tendinosis with promising results. Although similar effectiveness has been shown with sonographically guided tenotomy without corticosteroid injection in the elbow, to our knowledge, there are no similar studies assessing effectiveness in other tendons. ${ }^{16}$

We have incorporated the use of sonographic guidance in the treatment of recalcitrant tendon injuries but have avoided the administration of corticosteroids, fearing potential deleterious effects. ${ }^{17,18}$ The purpose of this study was to evaluate the safety and short-term effectiveness of sonographically guided needle tenotomy in the treatment of tendinosis.

\section{Materials and Methods}

Institutional Review Board approval was obtained. Patients were selected over an 18-month period from an outpatient university-based sports medicine clinic. Patients were included if the history and physical examination were consistent with a chronic tendon injury, followed by a sonographic examination identifying tendinosis. In most cases, additional imaging (eg, magnetic resonance imaging) was performed to exclude other diagnoses. Exclusion criteria were patients younger than 18 years, duration of symptoms less than 6 months, and patients with normal sonographic results. All patients had failed to respond to conservative treatment, including relative rest, pain medication, physical therapy emphasizing eccentric strengthening, braces, and, in 4 cases, "blind" corticosteroid injections without imaging guidance. All prior corticosteroid injections were at least 6 months before sonographically guided tenotomy. Informed consent was obtained from each patient before his or her entrance into the study. Each patient completed a questionnaire obtaining demographic data, the duration of symptoms, prior treatment, and a pain measurement using a visual analog scale (VAS). ${ }^{19}$

Sonographic examination for confirmation of diagnosis and for needle guidance was performed by one of the authors (J.A.J.), a fellowshiptrained musculoskeletal radiologist with 12 years of experience, using 7 - to $15-\mathrm{MHz}$ linear transducers (HDI 5000; Philips Healthcare, Bothell, WA). Liberal transmission gel was used in place of a standoff pad. A tendon was considered abnormal if the normal hyperechoic fibrillar echo texture was replaced with an abnormal hypoechoic or anechoic area. ${ }^{20}$ In addition, the abnormal tendon location was correlated with symptoms induced by direct transducer pressure. Soft tissue calcification or ossification was identified by its focal hyperechoic surface and variable posterior acoustic shadowing. Cortical irregularity was identified by its irregular echogenic contour. A correlation was made to color and power Doppler images when available. Using a sterile technique with a sterile probe cover, real-time sonographic guidance was provided for the percutaneous needling procedure.

For the tenotomy procedure, the needling was performed by a fellowship-trained sports medicine physician in conjunction with the fellowship-trained musculoskeletal radiologist who provided sonographic guidance; the use of 2 operators allowed continual sonographic guidance with accurate and precise needle placement. A sterile field was set up and maintained throughout the procedure. A 27-gauge needle (Becton Dickinson and Company, Franklin Lakes, NJ) was first used to anesthetize the skin with a 1:1 mixture of $1 \%$ lidocaine to $0.25 \%$ bupivacaine. The syringe was then switched over to a 22-gauge needle (Becton Dickinson and Company) to perform the tenotomy of the abnormal tendon. Approximately 20 to 30 needle passes were made through the abnormal region of the tendon with real-time sonographic imaging for continual 
guidance. If the tendon abnormality included the bone-tendon interface, this area was specifically targeted as well. The procedure was determined to be complete when the needle had been passed through all abnormal regions of the tendon (and enthesis) at least once. In each case, the needle was advanced along the long axis of the transducer only when it was visualized sonographically to ensure accurate needle placement. In 1 case, a 3.5-in, 22-gauge spinal needle (Becton Dickinson and Company) was used to reach the origin of the rectus femoris at an approximate depth of 7 to $8 \mathrm{~cm}$. During each procedure, approximately 3 to $6 \mathrm{~mL}$ of the lidocaine-bupivacaine mixture was injected into the tendon to maintain anesthesia. If calcifications were identified on sonography, these areas were targeted with the same 22-gauge needle to disrupt the calcifications.

The postprocedure protocol consisted of relative rest until the first follow-up appointment in 4 weeks. Patients were allowed to continue usual activities of daily living during that period regardless of an upper versus a lower extremity tenotomy site. A gradual return to activities was then discussed at the 4-week follow-up appointment. Physical therapy was not prescribed for the patients in this study after the procedure. All patients were evaluated at 4 weeks and all but 1 patient at 12 weeks after the procedure by a fellowship-trained sports medicine physician who recorded theirVAS scores and any complications.

Differences in the VAS pain scores between periods were assessed with a 1-way analysis of variance model. The type I probability was controlled at the $P<.05$ level. Pair-wise comparisons were assessed by the Tukey honestly significant difference post hoc test. The analysis was performed with Systat version 11 (Systat Software, Inc, Richmond, CA).

\section{Results}

A total of 14 tendons in 13 patients (9 male and 4 female) were entered into the study, with an average patient age of 38.5 years (range, 18-52 years). The average duration of symptoms was 53 months (range, 6-240 months). The specific tendons treated (with the total number in parentheses) were as follows: patellar (5; Figure 1), Achilles
(4), proximal gluteus medius (1; Figure 2), proximal iliotibial tract (1), proximal hamstring (1), common extensor elbow (1), and proximal rectus femoris (1). With regard to the Achilles tendon, the areas of abnormality were proximal in 2 and distal at the calcaneus insertion in 2.

No complications were noted during the procedure, or at the 4- and 12-week follow-up visits. Immediately after the procedure, there was the expected occurrence of minimal bleeding at the needle site. Bleeding was easily controlled with an adhesive bandage.

The mean pain rating scores \pm SEM at the baseline and 4 and 12 weeks were $5.8 \pm 0.6,2.4 \pm 0.7$, and $2.2 \pm 0.7$, respectively. We found a statistically significant difference $(P<.001)$ between mean pain scores at the baseline compared with both 4 and 12 weeks after treatment (Figure 3) No differences were observed between the 4- and 12week posttreatment data.

\section{Discussion}

The results of this study of selected patients with chronic tendinopathy showed that sonographically guided tenotomy had no complications and resulted in a statistically significant reduction in mean pain scores 4 weeks after the procedure. The pain reduction was maintained at the 12-week follow-up.

The concept of treating chronic tendon injuries with needling has a history in veterinary medicine by the descriptive term "pin-firing," a technique in which veterinarians used a firing iron to create a pattern of soft tissue burns in an attempt to change a chronic tendon injury into an acute inflammatory state (G. O'Connor, MD, oral communication, 2002). This is obviously a cruel method of treatment, which has been banned and (it is hoped) is no longer used. However, the idea of deliberately changing a chronic, nonhealing injury (such as occurs in tendinosis) into an acute condition that may have greater healing potential is intriguing and provided the impetus for this and other studies.

It is thought by some that the common denominator of tenotomy and needle injections is the disruption of tendinosis or scar tissue and the induction of bleeding, which in turn releases certain growth factors (those identified ${ }^{11,21}$ and 
those yet unidentified) that stimulate a healing response. This is consistent with a study by Taylor et $\mathrm{al}^{22}$ that showed increased strength of rabbit patellar tendons at 12 weeks after injection of autologous blood, a rich source of growth factors. Sonographically guided percutaneous tenotomy can target an abnormal tendon, including its bony attachment if needed.

The use of corticosteroid injections to treat tendinosis is debated. A recent study showed that percutaneous tenotomy with steroid injection was more effective than both percutaneous tenotomy with a local anesthetic and steroid injection alone; however, that study did not use sonographic guidance and only assessed the lateral epicondyle region of the elbow. ${ }^{23}$ Sonographic guidance provides pinpoint accuracy with percutaneous injections. An additional study using sonographic guidance showed that corticosteroids were not required for treatment of tennis elbow during percutaneous tenotomy. ${ }^{16}$ This is not unexpected given that clinical "tendinitis" has been shown to actually represent mucoid degeneration from repetitive microtrauma with a possible interstitial tendon tear rather than an acute inflammatory condition. ${ }^{16,24}$ In addition, because the positive effects of tenotomy are attributed to inciting a local inflammatory response, it is possible that corticosteroids may actually inhibit this process, and in fact, injection of corticosteroids is less effective when used long term. ${ }^{16}$

Although there is evidence that needling of tendinosis alone has a potential benefit, there are a number of injection therapies that are currently being investigated, which include prolotherapy, polidocanol, autologous whole blood, and platelet-rich plasma injections. ${ }^{25}$ Prolotherapy most commonly involves injection of hyperosmolar dextrose and morrhuate sodium, although the effects of the injection, whether inducing inflammation or causing sclerosis, are not clear. Polidocanol is a sclerosing agent that has been used to target neovascularity of tendinopathy

Figure 1. Images from a 36-year-old man with proximal patellar tendinosis and VAS scores of 6 (before the procedure), 1 (at 4 weeks), and 1 (at 12 weeks). A, Sonogram longitudinal to the proximal patellar tendon showing tendinosis as abnormal hypoechogenicity (arrows) with a normal distal patellar tendon (arrowheads). B, Sonogram transverse to the proximal patellar tendon showing abnormal hypoechogenicity (arrows) within the patellar tendon (arrowheads). C, Power Doppler sonogram in the sagittal plane showing increased flow. D, Sonogram longitudinal to the patellar tendon during tenotomy showing the echogenic 22-gauge needle (arrows) with the distal tip in the area of tendinosis. F indicates femur; and $\mathrm{P}$, patella.

A

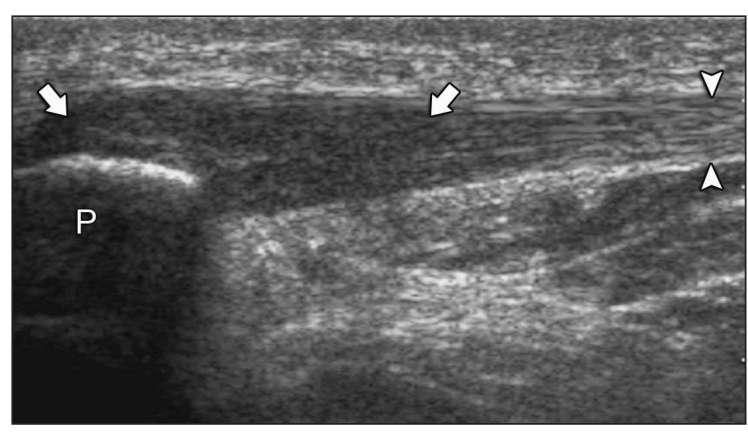

C

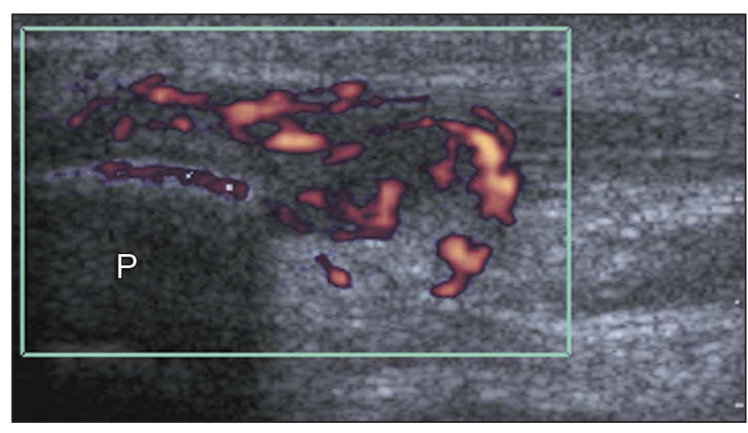

B

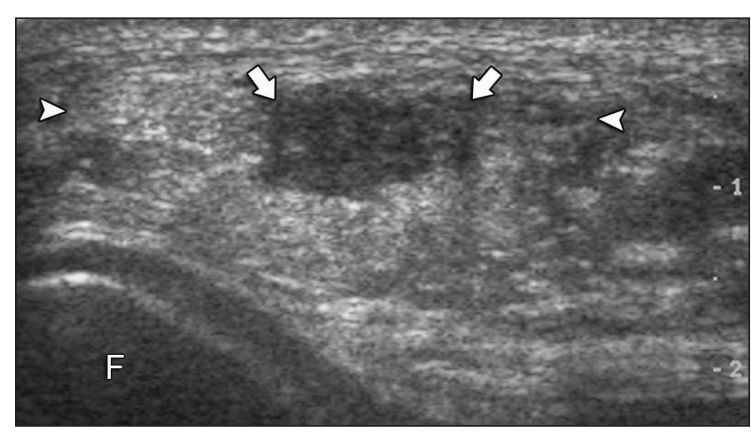

D

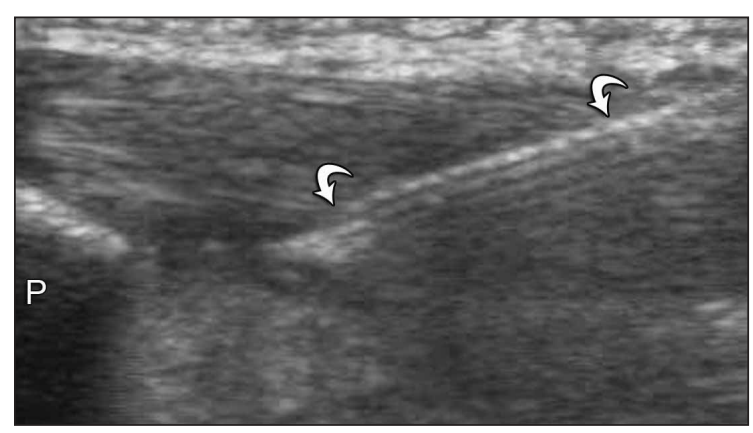


and has been used for the treatment of Achilles tendinopathy, ${ }^{26}$ patellar tendinopathy, ${ }^{27}$ and "tennis elbow," 28 although in the study with tennis elbow, there was no significant outcome difference in those injected with polidocanol and those injected with lidocaine and epinephrine. ${ }^{28}$ Both autologous whole blood and platelet-rich plasma are injected into areas of tendinopathy with the goal of inducing healing via cellular and humoral mediators. ${ }^{25}$ It is possible that percutaneous tenotomy without injection also produces healing via the same mechanism. Further studies comparing these various injection therapies to percutaneous needle tenotomy alone are needed.

The use of sonographic guidance in the treatment of musculoskeletal injuries is steadily gaining exposure in the literature..$^{9,10,26-30}$ Musculoskeletal sonography offers dynamic

Figure 2. Images from a 39-year-old man with proximal gluteus medius tendinosis and VAS scores of 6 (before the procedure), 0 (at 4 weeks), and 0 (at 12 weeks). A, Sonogram longitudinal to the proximal gluteus medius showing hypoechoic swelling (arrows) of the gluteus medius (Gmed) and overlying gluteal aponeurosis (arrowhead). B, Sonogram longitudinal to the gluteus medius during tenotomy showing the echogenic 22-gauge needle (arrows) with the distal tip in the area of tendinosis. I indicates ilium.

A

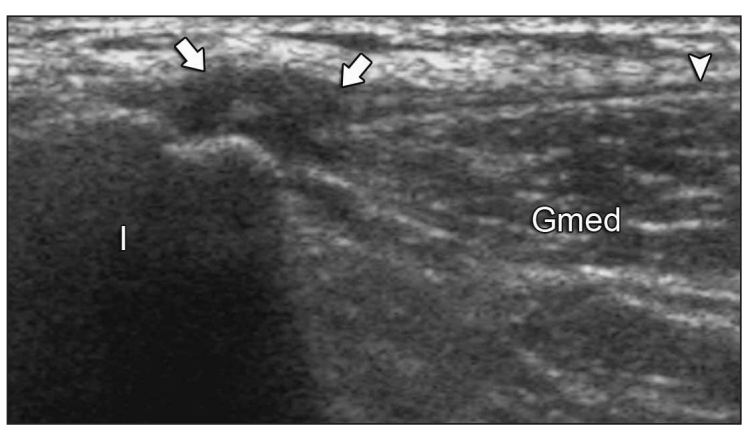

B

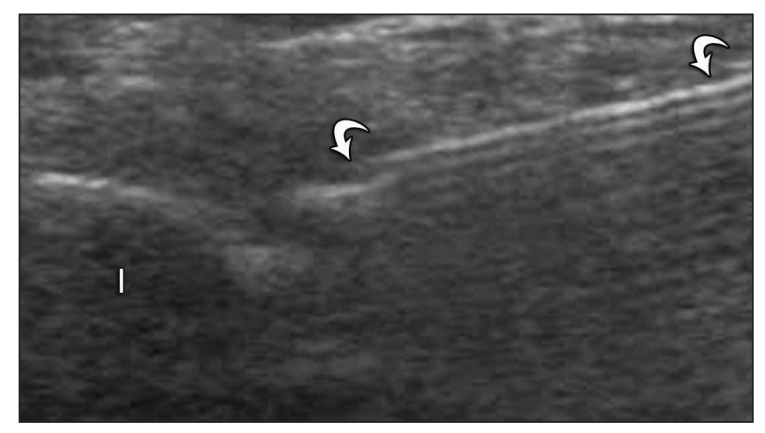

and real-time tendon imaging, ${ }^{20}$ with essentially no harmful effects to the patient. From a procedural standpoint, sonography is helpful both to diagnose the precise location of tendon abnormalities ${ }^{29}$ and to provide continual guidance regarding the needle location. ${ }^{31}$

We recognize that there were several limitations to this study. The study population was a relatively inhomogeneous group of patients because we wanted to report our experience with multiple tendons. In addition, previous clinical treatments were not standardized, although that parallels routine clinical practice. As another limitation, we did not compare our patients to a control group; the patients and investigators were not blinded; and results were obtained over a relatively short follow-up period. Last, sonographic follow-up was not performed, although on the basis of the work of Coleman et $\mathrm{al}^{2}$ and Khan et $\mathrm{al}^{7}{ }^{7}$ that would be of questionable benefit given the added expense and patient inconvenience. Thus, the results should be viewed in the context of these limitations.

The results of this study show that sonographically guided percutaneous needle tenotomy without corticosteroid injection is effective in reducing pain in patients with chronic tendinopathy without complications. Further investigation into the clinical utility of this procedure in a larger patient population with comparison to other treatment options for chronic tendinopathy is warranted.

Figure 3. Change in the mean VAS score over time.

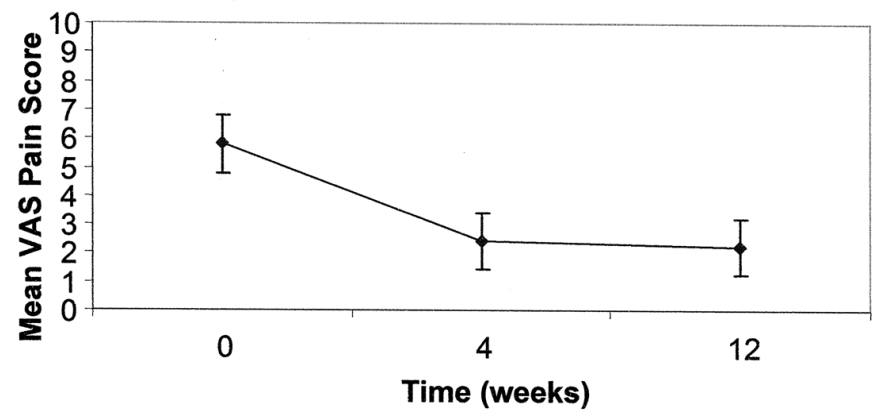




\section{References}

1. Khan K, Cook J. The painful nonruptured tendon: clinical aspects. Clin Sports Med 2003; 22:711-725.

2. Coleman BD, Khan KM, Kiss ZS, Bartlett J, Young DA, Wark JD. Open and arthroscopic patellar tenotomy for chronic patellar tendinopathy: a retrospective outcome study. Victorian Institute of Sport Tendon Study Group. Am J Sports Med 2000; 28:183-190.

3. Coleman BD, Khan KM, Maffulli N, Cook JL, Wark JD. Studies of surgical outcome after patellar tendinopathy: clinical significance of methodological deficiencies and guidelines for future studies. Victorian Institute of Sport Tendon Study Group. Scand J Med Sci Sports 2000; 10:2-11.

4. Leadbetter WB, Mooar PA, Lane GJ, Lee SJ. The surgical treatment of tendinitis: clinical rationale and biologic basis. Clin Sports Med 1992; 11:679-712.

5. Tallon C, Coleman BD, Khan KM, Maffulli N. Outcome of surgery for chronic Achilles tendinopathy: a critical review. Am J Sports Med 2001; 29:315-320.

6. Paavola $M$, Orava $S$, Leppilahti J, Kannus $P$, Järvinen $M$ Chronic Achilles tendon overuse injury: complications after surgical treatment. An analysis of 432 consecutive patients. Am J Sports Med 2000; 28:77-82.

7. Khan KM, Visentini PJ, Kiss ZS, et al. Correlation of ultrasound and magnetic resonance imaging with clinical outcome after patellar tenotomy: prospective and retrospective studies. Victorian Institute of Sport Tendon Study Group. Clin I Sport Med 1999; 9:129-137.

8. Sandmeier R, Renström PA. Diagnosis and treatment of chronic tendon disorders in sports. Scand J Med Sci Sports 1997; 7:96-106.

9. Testa V, Capasso G, Benazzo F, Maffulli N. Management of Achilles tendinopathy by ultrasound-guided percutaneous tenotomy. Med Sci Sports Exerc 2002; 34:573-580.

10. Testa V, Capasso G, Maffulli N, Bifulco G. Ultrasound-guided percutaneous longitudinal tenotomy for the management of patellar tendinopathy. Med Sci Sports Exerc 1999; 31:1509_ 1515.

11. Buchbinder R, Ptasznik R, Gordon J, Buchanan J, Prabaharan $V$, Forbes A. Ultrasound-guided extracorporeal shock wave therapy for plantar fasciitis: a randomized controlled trial. JAMA 2002; 288:1364-1372.

12. Charrin JE, Noël ER. Shockwave therapy under ultrasonographic guidance in rotator cuff calcific tendinitis. Joint Bone Spine 2001; 68:241-244.

13. Hyer $C F$, Vancourt $R$, Block A. Evaluation of ultrasound-guided extracorporeal shock wave therapy (ESWT) in the treatment of chronic plantar fasciitis. J Foot Ankle Surg 2005; 44:137-143.

14. Tasto JP, Eves WC, Valeu R, Winters W. Treatment of chronic tendinosis with bipolar radiofrequency stimulation. Paper presented at: Arthroscopy Association of North America Annual Meeting; 2003; Phoenix, AZ.

15. McShane JM, Nazarian LN, Harwood MI. Sonographically guided percutaneous needle tenotomy for treatment of com- mon extensor tendinosis in the elbow. J Ultrasound Med 2006; 25:1281-1289.

16. McShane JM, Shah VN, Nazarian LN. Sonographically guided percutaneous needle tenotomy for treatment of common extensor tendinosis in the elbow: is a corticosteroid necessary? J Ultrasound Med 2008; 27:1137-1144.

17. Anastassiades T, Dziewiatkowski D. The effect of cortisone on the metabolism of connective tissues in the rat. J Lab Clin Med 1970; 75:826-839.

18. Fredberg $U$. Local corticosteroid injection in sport: review of literature and guidelines for treatment. Scand J Med Sci Sports 1997; 7:131-139.

19. Miller MD, Ferris DG. Measurement of subjective phenomena in primary care research: the Visual Analogue Scale. Fam Pract Res J 1993; 13:15-24

20. Jacobson JA. Ultrasound in sports medicine. Radiol Clin North Am 2002; 40:363-386.

21. Hildebrand KA, Woo SL, Smith DW, et al. The effects of platelet-derived growth factor-BB on healing of the rabbit medial collateral ligament: an in vivo study. Am J Sports Med 1998; 26:549-554.

22. Taylor MA, Norman TL, Clovis NB, Blaha JD. The response of rabbit patellar tendons after autologous blood injection. Med Sci Sports Exerc 2002; 34:70-73.

23. Dogramaci $Y$, Kalaci $A$, Savas $N$, Duman IG, Yanat $A N$. Treatment of lateral epicondilitis using three different local injection modalities: a randomized prospective clinical trial. Arch Orthop Trauma Surg 2009. http://www.springerlink. com/content/a6v56436g6130q87/.

24. $\mathrm{Xu} Y$, Murrell GA. The basic science of tendinopathy. Clin Orthop Relat Res 2008; 466:1528-1538.

25. Rabago D, Best TM, Zgierska AE, Zeisig E, Ryan M, Crane D. A systematic review of four injection therapies for lateral epicondylosis: prolotherapy, polidocanol, whole blood and platelet-rich plasma. Br J Sports Med 2009; 43:471-481.

26. Alfredson $\mathrm{H}$, Ohberg L. Sclerosing injections to areas of neovascularisation reduce pain in chronic Achilles tendinopathy: a double-blind randomised controlled trial. Knee Surg Sports Traumatol Arthrosc 2005; 13:338-344.

27. Hoksrud A, Ohberg L, Alfredson H, Bahr R. Ultrasound-guided sclerosis of neovessels in painful chronic patellar tendinopathy: a randomized controlled trial. Am J Sports Med 2006; 34:1738-1746

28. Zeisig E, Fahlström M, Ohberg L, Alfredson H. Pain relief after intratendinous injections in patients with tennis elbow: results of a randomised study. Br J Sports Med 2008; 42:267-271.

29. Jacobson JA, van Holsbeeck MT. Musculoskeletal ultrasonography. Orthop Clin North Am 1998; 29:135-167.

30. Kane D, Greaney T, Bresnihan B, Gibney R, FitzGerald O. Ultrasound guided injection of recalcitrant plantar fasciitis. Ann Rheum Dis 1998; 57:383-384.

31. Cardinal E, Chhem RK, Beauregard CG. Ultrasound-guided interventional procedures in the musculoskeletal system. Radiol Clin North Am 1998; 36:597-604. 